\title{
Tests of Symmetries with $\eta$ Decays at WASA-at-COSY
}

\section{Daniel Coderre*}

for the WASA-at-COSY Collaboration

E-mail: d.coderreafz-juelich.de

\begin{abstract}
WASA-at-COSY is a hadron collision experiment located at Forschungszentrum Jülich, Germany. One focus of the experiment is the investigation of symmetry-breaking patterns in $\eta$-meson decays. A sample of $30 \times 10^{6} \eta$-mesons have been reconstructed from the $p d \rightarrow{ }^{3} \mathrm{He} \eta$ production reaction. Results from this data sample are presented here. An additional sample of $10^{9} \eta$-mesons produced in the $p p \rightarrow p p \eta$ reaction is currently undergoing analysis.

The decays $\eta \rightarrow \pi^{+} \pi^{-} \gamma$ and $\eta \rightarrow \pi^{+} \pi^{-} e^{+} e^{-}$proceed via anomalous terms in the QCD action. Measurements of these channels can help fix the magnitude of the leading-order contributions to the decay amplitude and determine the influence of final state interactions. WASA-at-COSY has previously published results on the relevant kinematic spectra in $\eta \rightarrow \pi^{+} \pi^{-} \gamma$. In this work, $263 \pm$ $24_{\text {stat }}$ events from the rare process $\eta \rightarrow \pi^{+} \pi^{-} e^{+} e^{-}$have been identified. Based on this event sample, the branching ratio has been extracted and an observable for possible flavor-conserving CP-violation has been investigated.
\end{abstract}

The 7th International Workshop on Chiral Dynamics,

August 6-10, 2012

Jefferson Lab, Newport News, Virginia, USA

${ }^{*}$ Forschungszentrum Jülich 


\section{The WASA-at-COSY Experiment}

The WASA-at-COSY experiment is a near $4-\pi$ detector installed internally at the Cooler Synchnotron (COSY) storage ring at Forschungszentrum Jülich, Germany [1]. A proton beam is impinged on a hydrogen or deuterium pellet target at energies just above the $\eta$-meson production threshold. A schematic diagram of the WASA-at-COSY detector is shown in Figure 1. A forward spectrometer covers the range from $3 \leq \theta \leq 18$ degrees in polar angle and is designed to stop and measure hadronic ejectiles. Particle identification and kinetic energy reconstruction for protons and helium nuclei are performed based on the ionization loss patterns in several layers of scintillators. Charged particle tracking is performed using a multi-wire proportional chamber consisting of 16 layers of straws arranged downstream from the exit window.

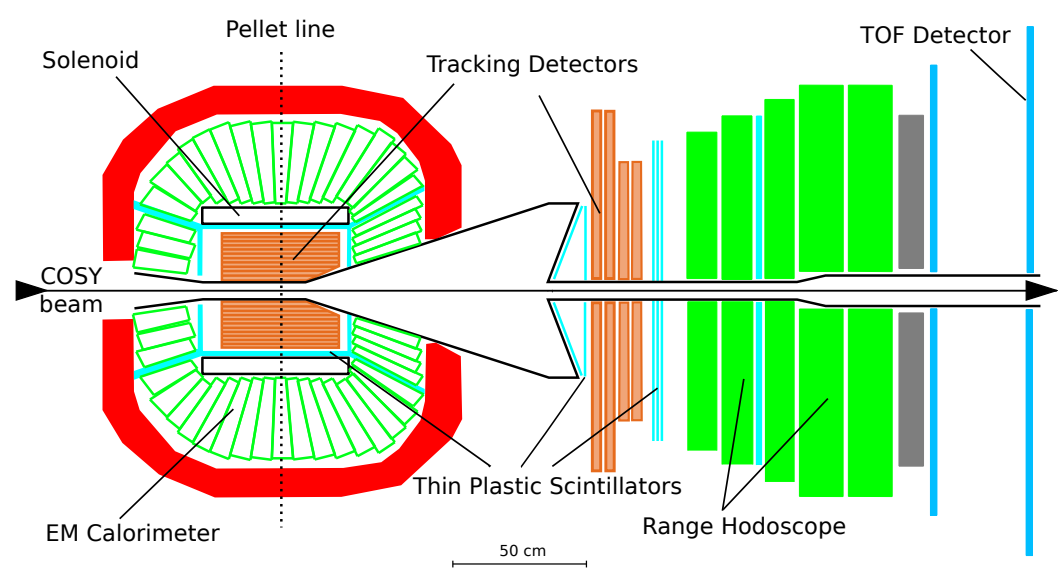

Figure 1: The WASA-at-COSY detector.

Meson decay products are measured by the central detector, which has acceptance of $20 \leq \theta \leq$ 160 degrees. The central detector is capable of momentum reconstruction of charged particles and photons. Directly surrounding the interaction region is a cylindrical drift chamber sitting within a homogeneous $1 \mathrm{~T}$ magnetic field parallel to the beam direction. The sign of a particle's charge and its momentum are determined respectively by the direction and radius of curvature of the particle track in the magnetic field. Directly surrounding the drift chamber, a layer of thin plastic scintillators provide a time reference for drift-time calibration and are used to distinguish charged $\pi$-mesons from electrons. The 1012-element $\mathrm{CsI}(\mathrm{Na})$ electromagnetic calorimeter surrounds the entire apparatus and is used for the measurement of photons. The calorimeter is additionally used to identify charged particles.

$\eta$-mesons at WASA-at-COSY are measured in both the $p d \rightarrow{ }^{3} \mathrm{He} \eta$ and $p p \rightarrow p p \eta$ reactions. In the proton-deutron data, the unique signal left by the helium ion allows efficient tagging of $p d \rightarrow{ }^{3} \mathrm{HeX}$ events even on the trigger level. This effectively filters out the vast majority of the total proton-deuteron cross section and allows the experiment to be run with no trigger condition on the decay of the $\eta$. Within the subset of data where a helium ion is present, the ratio of the cross-section of $\eta$ production to the cross section of multi-pion production is favorable, allowing signals from the $\eta$ to be seen without any analysis conditions. Due to hardware limitations on the 
maximum luminosity, the rate of $\eta$ production in proton-deuteron reactions is limited to about 10 $\eta$-mesons per second.

In proton-proton reactions, a higher production cross second allows a rate of over $100 \eta$ mesons per second to be reached. However, backgrounds from multi-pion production necessitate a trigger biased towards specific decays of the $\eta$. The larger backgrounds also demand extra analysis steps in order to observe clean signals from $\eta$ mesons. An average increase of statistics of at least a factor of five over $\mathrm{p}-\mathrm{d}$ can be achieved in this data.

The analysis presented here is based on an event sample of $3 \times 10^{6}$ reconstructed $\eta$-mesons from the proton-deuteron data. A much larger data set consising of over $5 \times 10^{8}$ produced $\eta$ mesons has been collected in the proton-proton reaction and is in the early stages of analysis. The WASA-at-COSY collaboration is measuring several final states as part of its $\eta$ decay program. The analysis of the rare decay $\eta \rightarrow \pi^{+} \pi^{-} e^{+} e^{-}$is presented here.

\section{Measurement of the Rare Channel $\eta \rightarrow \pi^{+} \pi^{-} e^{+} e^{-}$}

The channels $\eta \rightarrow \pi^{+} \pi^{-} \gamma$ and $\eta \rightarrow \pi^{+} \pi^{-} e^{+} e^{-}$are based on the same underlying mechanisms and differ only by the real (virtual) photon in the final state. At the chiral limit, these reactions proceed exclusively through the box-anomaly term of the Wess-Zumino-Witten Lagrangian, which describes the coupling of a pseudoscalar to two pseudoscalars and a vector[2,3]. In principle these decays should allow study of the box-anomaly. This is however not straightforward, since the signal from the box diagram is obscurred by contributions of additional mechanisms containing intermediate vector-meson states and final state interactions $[5,6,7,8]$.

In order to provide more information with which to disentangle the various contributions to the decay amplitude, experiments can measure the kinematic distributions of the final state particles as well as the branching ratios of the decays. The kinematic distributions have been measured by both the WASA-at-COSY and KLOE collaborations [4, 9]. The branching ratio has recently become controversial due to a pair of measurements of $\eta \rightarrow \pi^{+} \pi^{-} \gamma$ from the CLEO and KLOE collaborations which reported a value about three standard deviations lower than the former world average [10,9]. Since the channel $\eta \rightarrow \pi^{+} \pi^{-} e^{+} e^{-}$is directly related, measurements can be made in both final states and must provide consistent results. WASA-at-COSY is currently extracting a result for the branching ratio of both channels. The analysis of $\eta \rightarrow \pi^{+} \pi^{-} e^{+} e^{-}$is described here.

The channel $\eta \rightarrow \pi^{+} \pi^{-} e^{+} e^{-}$is also interesting because it contains a possible observable for CP-violation outside the Standard Model [11, 12]. It has been suggested that if the CP-violating E1 transition contributes to the decay amplitude this would lead to an asymmetry in the electron and pion decay planes in the final state particles. There is no mechanism in the Standard Model that would explain such CP-violation, but it is possible to derive an operator through which the reaction could proceed without violating any existing experimental constraints [11, 12]. Using this operator, a theoretical upper limit of $1 \%$ has been calculated. Because this channel is strongly suppressed, with a branching ratio of $(2.68 \pm 0.11) \times 10^{-4}$, measurements of this asymmetry have only been attempted in recent years [13]. The current experimental value for the asymmetry term, based on one measurement, is $-0.006 \pm 0.031$ [14].

At WASA-at-COSY, all $\eta \rightarrow \pi^{+} \pi^{-} e^{+} e^{-}$events are required to have a ${ }^{3} \mathrm{He}$ ion reconstructed in the forward detector and four particles with proper charge reconstructed in the drift chamber. In 
order to distinguish the particles as electrons or $\pi$-mesons, the signed momentum from the drift chamber is graphed against the energy a particle deposits in the plastic scintillator detectors and the calorimeter. These energy-loss patterns form distinct bands corresponding to pions and electrons, as shown in Figure 2a for the calorimeter. To quantify this information, simulations of pure samples of pions and electrons are made and trained into neural networks. The neural networks are then used at run time to provide a probability that a given reconstructed particle is a pion or electron. The opening angle between particles with opposite charged is also used for identification, with the dilepton pair having the smallest opening angle in over $80 \%$ of cases. Information from the neural networks for all particles is combined with the probability based on the opening angles using Bayes' equation. After all information is considered, the identification configuration with the highest probability is taken. Tests on simulations show the neural network based algoritm is over 95\% effective in assigning the correct particle types in $\eta \rightarrow \pi^{+} \pi^{-} e^{+} e^{-}$events.

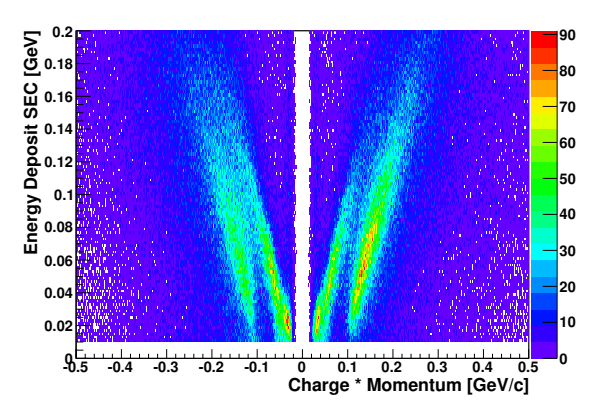

(a) Particle identification bands

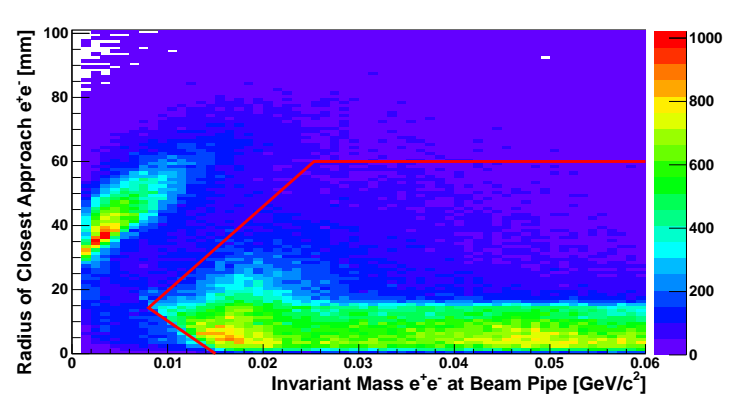

(b) Conversion condition

Figure 2: Identification bands used to separate electrons from pions are shown in Figure 2a. The condition used to reject dilepton pairs coming from conversion of photons in the detector material is shown in Figure 2b.

Accurate particle identification is required in order to assign masses for the kinematic fit. Only events passing a $\chi^{2}$ condition on the fit, which enforces total energy and momentum conservation only, are accepted. After the kinematic fit the event sample still contains background from events where the $e^{+} e^{-}$pair originates from a photon conversion in the beam pipe. These events are kinematically extremely similar to the signal channel and can come from, for example, $\eta \rightarrow \pi^{+} \pi^{-} \gamma$ events. To suppress these events, a vertex position is calculated by determining the closest approach of the $e^{+} e^{-}$helices. The distance of this vertex to the origin (interaction point) is graphed against the invariant mass of the dilepton pair recalculated at the point where the particle passes through the beam pipe. This provides a clean separation of conversion events from events originating at the origin, as shown in Figure 2b.

After all selection criteria, the invariant mass of the remaining event candidates is graphed, as shown in Figure 3a. The background from non-resonant pion production is fitted with a polynomial and subtracted. Based on simulations, the remaining $\eta$ events are still about $35 \%$ background with the dominant contribution coming from the channel $\eta \rightarrow \pi^{+} \pi^{-}\left[\pi^{0} \rightarrow e^{+} e^{-} \gamma\right]$, where the final state differs by a single low-energy photon. After subtracting the background from other $\eta$ channels, $263 \pm 24_{\text {stat }}$ events remain and are attributed to the signal channel. The branching ratio is calculated 


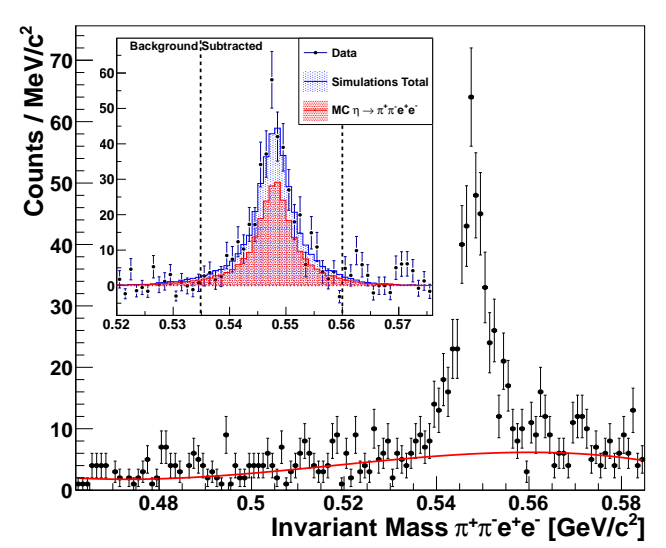

(a) Events surviving all conditions

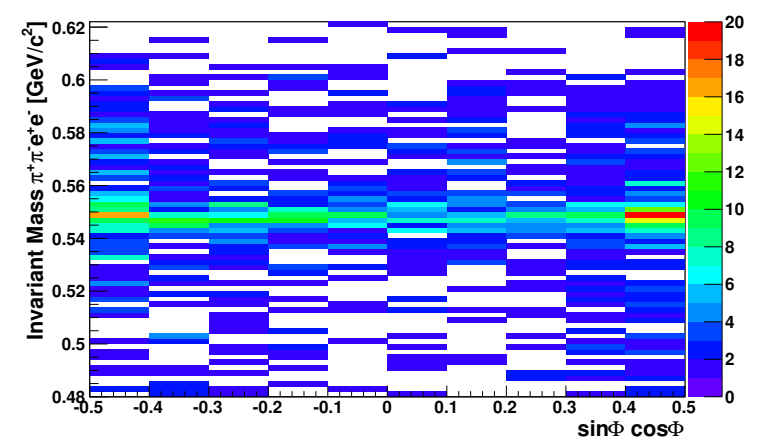

(b) Invariant mass versus dihedral angle

Figure 3: Figure 3a shows the final $\eta \rightarrow \pi^{+} \pi^{-} e^{+} e^{-}$event candidates after all selection conditions. The invariant mass versus dihedral angle is shown in Figure $3 b$.

using $666 \pm 30_{\text {stat }}$ events identified from the channel $\eta \rightarrow \pi^{+} \pi^{-}\left[\pi^{0} \rightarrow e^{+} e^{-} \gamma\right]$ for normalization. This final state is selected using the exact same conditions as the signal channel except for the hypothesis for the kinematic fit. Due to its unique decay signature and higher branching ratio, it can be selected with over $90 \%$ purity. Normalizing to such a similar final state allows several sources of systematic error to cancel out. Using the branching ratios of the normalization decay chain from Ref. [13], a preliminary value for the branching ratio of the signal channel has been extracted [15].

$$
B R\left(\eta \rightarrow \pi^{+} \pi^{-} e^{+} e^{-}\right)=\left(3.10 \pm 0.27_{\text {stat }} \pm 0.22_{\text {sys }}\right) \times 10^{-4}
$$

To extract the asymmetry term, the invariant mass of each event candidate was graphed against the dihedral angle as shown in Figure 3b. The statistics are sufficient for two projections, for $\sin \phi \cos \phi$ greater than or less than zero. The continuum pion background is subtracted with a polynomial and the remaining events are counted. After subtraction of $\eta$ background, which was verified in simulations to exhibit no asymmetry, the preliminary value for the asymmetry term was determined [15].

$$
A_{\phi}=\left(0.4 \pm 9.0_{\text {stat }} \pm 2.8_{\text {sys }}\right) \times 10^{-2}
$$

Both of these values are preliminary and are undergoing final systematic checks. The asymmetry term in particular is greatly limited by the available statistics. Analysis of WASA-at-COSY data in proton-proton reactions should allow the statistical error on this measurement to be reduced by at least a factor of three. 


\section{References}

[1] H. -H. Adam et al. [WASA-at-COSY Collaboration], nucl-ex/0411038.

[2] J. Wess and B. Zumino, Phys. Lett. B 37, 95-97 (1971).

[3] E.Witten, Nucl. Phys. B 223, 422-432 (1983).

[4] P. Adlarson et al. [WASA-at-COSY Collaboration], Phys. Lett. B 707, 243-249 (2012).

[5] C. Piccioto and S. Eichardson, Phys. Rev. D 48, 3395-3396 (1993).

[6] A. Faessler, C. Fuchs, and M. Krivoruchenko, Phys. Rev. C 61, 035206 (2000).

[7] B. Borasoy and R. Nissler, Eur. Phys. J. A 33, 95-106 (2007).

[8] T. Petri, Master's Thesis, Rheinischen Friedrich-Wellhelms Universität Bonn, October 2010, arXiv:nucl-th/1010.2378.

[9] D. Babusci et al. [KLOE Collaboration], Phys. Lett. B 718, 910-914 (2013).

[10] A. Lopex et al. [CLEO Collaboration], Phys. Rev. Lett. 99, 122001 (2007).

[11] D. Gao, Mod. Phys. Lett. A 17, 1583-1588 (2002).

[12] C.Geng, J. Ng, and T. Wu, Mod. Phys. Lett. A 17, 1489-1497 (2002).

[13] J. Beringer et al. [Particle Data Group], Phys. Rev. D 86, 010001 (2012).

[14] F. Ambrosino et al. [KLOE Collaboration], Phys. Lett. B 675, 283-288 (2009).

[15] D. Coderre, Doctoral Thesis, Ruhr-Universität Bochum. (2012). 